\title{
53. TRES NUEVOS HÍBRIDOS PARA EL GÉNERO TEUCRIUM L. SECCIÓN POLIUM (MILL.) SCHREB. (LAMIACEAE) EN EL SUDESTE IBÉRICO.
}

\author{
Pedro SÁNCHEZ-GÓMEZ, Jaime GÜEMES, A. Félix CARRILLO, \\ Ernesto COY y Antonio HERNÁNDEZ
}

Three new hybrids for the genus Teucrium L. section Polium (Mill.) Schreb. (Lamiaceae) in the south-eastern Spain.

Palabras clave: Híbridos, Teucrium, Sudeste Ibérico.

Key words: Hybrids, Teucrium, Southeast of Spain.

A partir de los últimos trabajos relativos al género Teucrium L. sección Polium (Mill.) Schreb. (Puech, 1984; Navarro, 1988, 1995; Navarro \& Rosúa, 1990) se han aportado datos precisos relativos a taxones hasta la fecha poco conocidos o confusos, que, a su vez, han permitido reconocer y describir buen número de nototaxones. Como resultado de las investigaciones que sobre la flora de sudeste Ibérico vienen realizándose, se han detectado tres nuevos híbridos interespecíficos del género Teucrium sección Polium, dos entre taxones de la subsección Rotundifolia Cohen ex Valdés Berm. \& Sánchez Crespo y el restante para la subsección Polium (Mill.) Kästner que son descritos a continuación:

\section{Teucrium x pseudothymifolium Sánchez-}

Gómez, J. Güemes \& A.F. Carrillo nothosp.nov. T. rotundifolium Schreb. $\mathrm{x}$ T. thymifolium Schreb.

Planta intermedia inter parentes. A $T$. rotundifolio differt foliis 2-3 crenatis oblongolanceolatis, cuneiformiis numquam cum basi subrotundata. A T. thymifolio differt indumento pilis hirsutis, calice pilis glandulosis.

Holotypus: MUB 20671 (sub T. buxifolium). Murcia; Moratalla, Arroyo de Hondares. 1000 m. 25-5-1983. 30SWH8831. Roquedos calizos. C. Selma.
Planta de hasta $5 \mathrm{~cm}$, cespitosa, villosa. Hojas (2,8) 3,8 x (4,5) 5,5 mm oblongolanceoladas de base cuneada con 2-3 crenaciones en el ápice. Brácteas y bracteolas semejantes a las hojas. Inflorescencia en cabezuela terminal pauciflora. Cáliz de 5,5-7 mm, tubular-campanulado con dientes ligeramente agudos, indumento de pelos sencillos, glandulares. Corola 8,5-10 mm, blanquecina.

La presencia de introgresiones y formas intermedias entre los parentales en los roquedos de las sierras béticas del Noroeste murciano y Suroeste albaceteño, única zona donde conviven los parentales, ya había sido puesta de manifiesto (Sánchez-Gómez \& Alcaraz, 1993: 215).

La planta presenta características intermedias entre los parentales, que son frecuentes en la localidad mencionada. Las hojas caulinares suelen ser oblongolanceoladas y generalmente cuneiformes, nunca suborbiculares 0 subrotundas $(T$. rotundifolium) ni oval-lanceoladas ( $T$. thymifolium); presentan solo 2-3 crenaciones laterales en el ápice, carácter más próximo a $T$. thymifolium no siendo crenado-lobuladas en todo su contorno ( $T$. rotundifolium). El cáliz (5,5-7mm) es de tamaño intermedio al de los parentales, tubular-campanulado, con un indumento hirsuto de pelos largos, sobre todo 

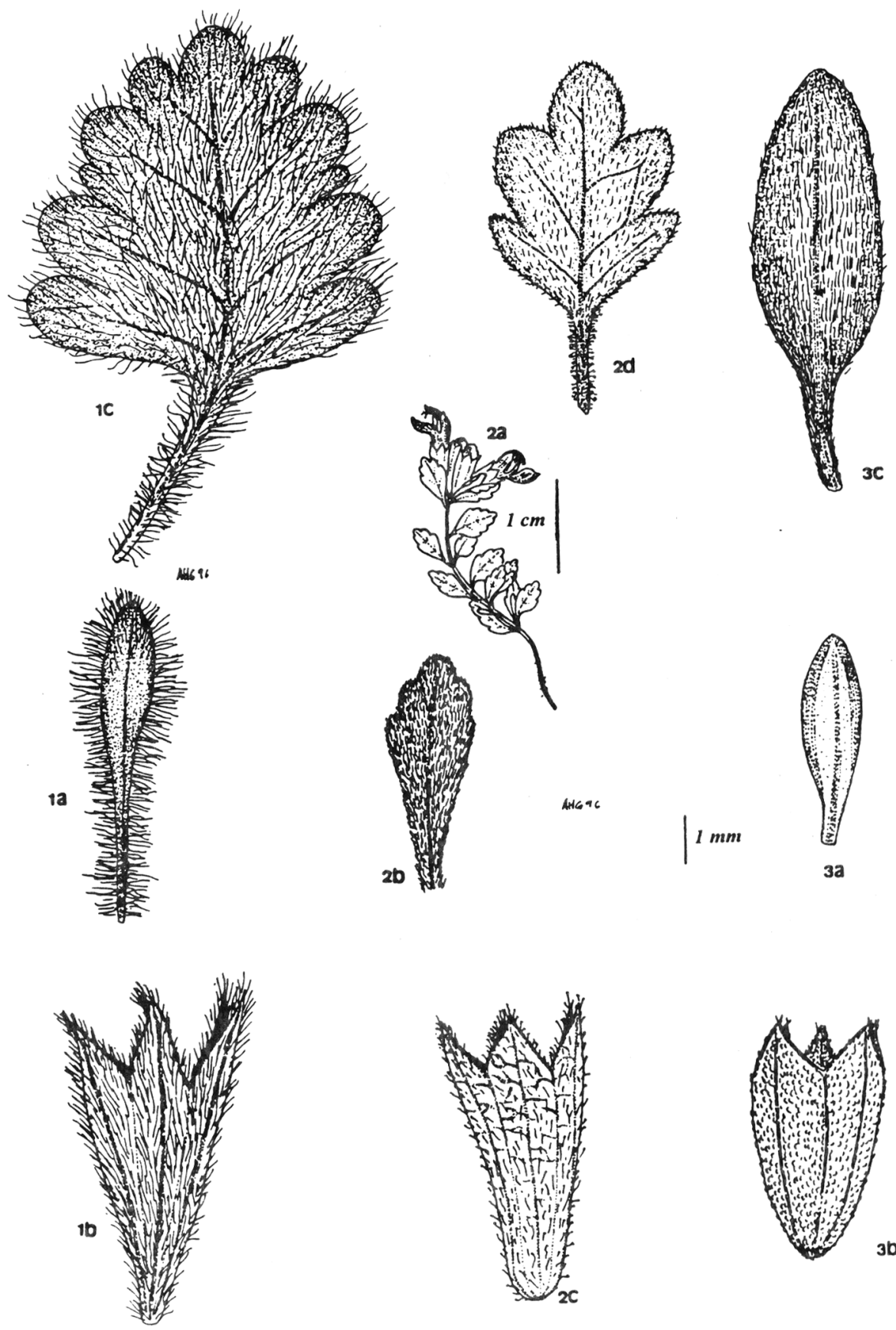

Figura 1. 1.- Teucrium rotundifolium: a) bracteola; b) cáliz; c) hoja. 2.- Teucrium x pseudothymifolium: a) hábito; b) bracteola; c) cáliz; d) hoja. 3.- Teucrium thymifolium: a) bracteola; b) cáliz; c) hoja. 1.- Teucrium rotundifolium: a) floral leave; b) calyx; c) leave. 2.- Teucrium x pseudothymifolium: a) habit; b) floral leave; c) calyx; d) leave. 3.- Teucrium thymifolium: a) floral leave; b) calyx; c) leave. 
glandulares, más largos y patentes que en $T$. thymifolium, pero más laxamente dispuestos y cortos que los de $T$. rotundifolium (fig. 1).

Teucrium x navarroi Sánchez-Gómez, J. Güemes, A. F. Carrillo, E. Coy \& A. Hernández nothosp nov.

T. rivas-martinezii Alcaraz, Garre, Mart. Parras \& Peinado x T. thymifolium Schreb.

Planta robusta quasi intermedia inter parentes. A T rivas-martinezii differt caulibus brevioribus numquam erecto-ascendentibus saepe foliis apice crenato, calice tubularcampanulatis numquam tubulatis. T. thymifolio simile in habito a quo tamen differt foliis maioribus 6-8,5×2,5-3,5 mm, calice maiore $(6,5-8 \mathrm{~mm})$ tubular-campanulato.

Dedicada a la Dra. Teresa Navarro.

Holotypus: MUB: 47535. Murcia; Calasparra, Embalse de Alfonso XIII. 30SWH2231. $350 \mathrm{~m}$. Roquedos calizos verticales. P. Sánchez-Gómez, J. Guemes \& A.F. Carrillo. Isotyus: MGC 42000; VAL 35854 .

Planta de hasta $30 \mathrm{~cm}$, ramas decumbentes, intrincadas, las florales ascendentes a erectas. Hojas (6) 8,5x(2,5)3,5 mm, oval-lanceoladas a oblongo-lanceoladas, grisáceas, a veces crenadas en el ápice, margen entero o poco revoluto. Brácteas $9 \times 3,5 \mathrm{~mm}$, romboideas, agudas y de márgenes enteros. Bracteolas con pelos glandulares. Inflorescencia en cabezuelas terminales de (8)15-25 flores. Cáliz 6,5-8 mm, tubular-campanulado con dientes triangulares, ligeramente agudos e indumento de pelos glandulares de hasta $1 \mathrm{~mm}$. Corola 10-12 mm, blanquecina con lóbulos latero-posteriores largos.

La presencia de este híbrido e introgresiones entre $T$. thymifolium y $T$. rivasmartinezii ya había sido puesta de manifiesto en las sierras de Segura Orientales (Sánchez-
Gómez \& Alcaraz, 1993: 214-215). T. rivasmartinezii es una especie poco conocida recientemente descrita. Estudios sobre su areal y morfología han puesto de manifiesto una amplia variabilidad, desde las formas suberectas, con cálices de pelos adpresos y no glandulares, que se circunscriben a la parte occidental del areal y que recuerdan vagamente a $T$. thymifolium, hasta las formas erectoascendentes, caulescentes, con cálices de pelos glandulares, simples y claviformes, frecuentes en la zona de Calasparra (localidad clásica).

En las proximidades de la localidad clásica conviven en los roquedos $T$. rivas-martinezii con formas particulares de T. thymifolium de hojas anchamente ovales y cálices relativamente grandes con indumento denso de pelos más largos. Entre ambos se presentan ejemplares claramente intermedios de considerable vigor híbrido.

T. x navarroi presenta tallos florales más cortos que los de $T$. rivas-martinezii. Los cálices, de hasta $8 \mathrm{~mm}$ son parecidos a los de $T$. rivas-martinezii, pero son tubularcampanulados, no tubulares, ni ligeramente gibosos en la base, además no presentan indumento glandular. La corola tiene los lóbulos lateroposteriores muy largos, carácter más próximo a $T$. rivas-martinezii; las bracteolas poseen pequeños pelos glandulares, ausentes en $T$. thymifolium. Las hojas y brácteas florales son claramente intermedias tanto en forma como en tamaño, algunas de ellas con crenaciones en el ápice, como en $T$. thymifolium (fig. 2).

Teucrium x conquense M.B. Crespo \& Mateo nothosubsp. siyasense A. F. Carrillo \& Sánchez-Gómez nothosubsp. nov.

T. capitatum subsp. gracillimum (Rouy) Valdés Berm. \& Sánchez Crespo x $T$. gnaphalodes L'Hér.

T. capitato subsp. gracillimo similis calice 


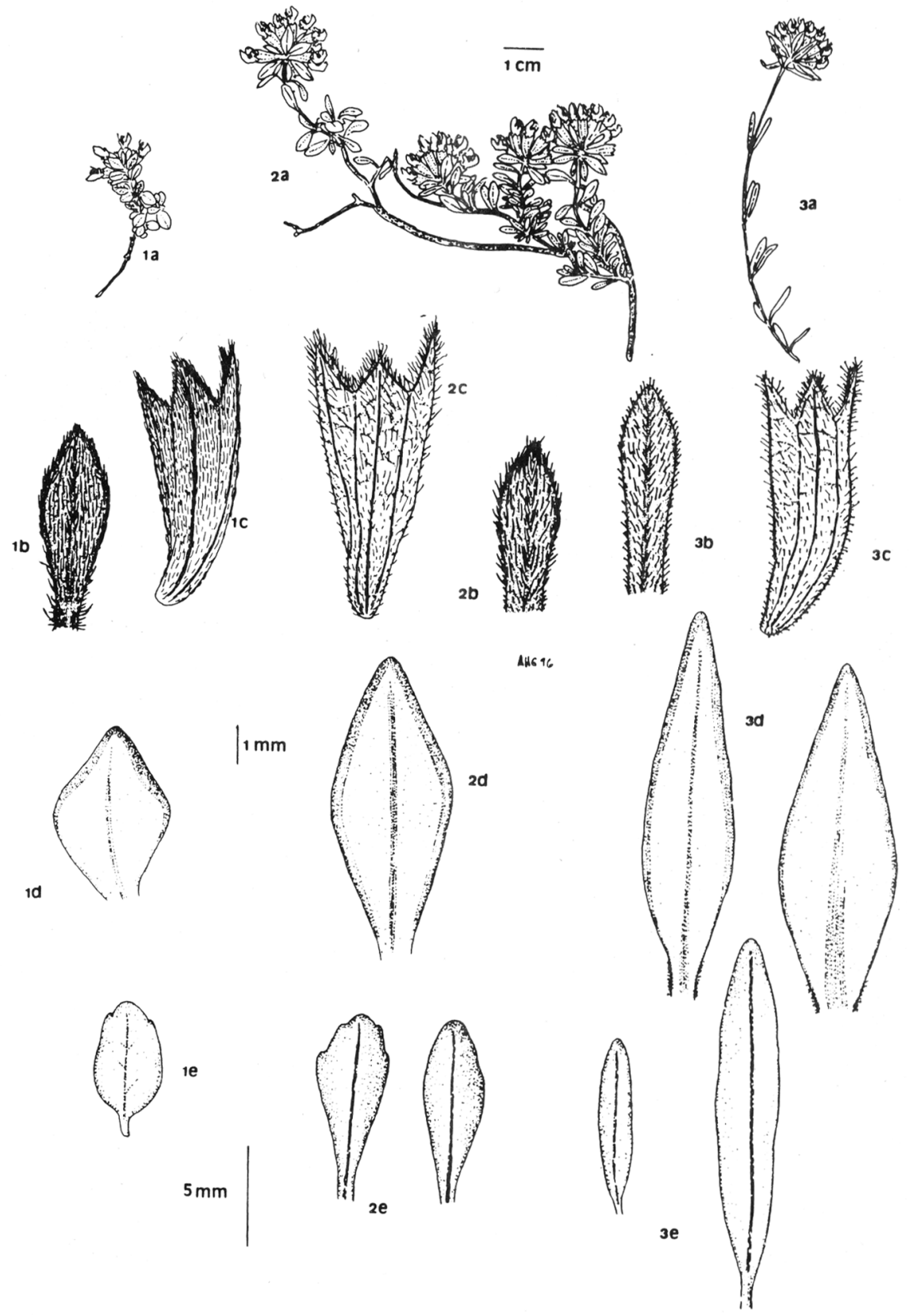

Figura 2. 1.-Teucrium thymifolium: a) rama e inflorescencia; b) bracteola; c) cáliz; d) bráctea; e) hoja. 2.Teucrium x navarroi: a) hábito; b) bracteola; c) cáliz; d) bráctea; e) hoja. 3.-Teucrium rivas-martinezii: a) rama e inflorescencia; b) bracteola; c) cáliz; d) brácteas; e) hojas. 1.-Teucrium thymifolium: a) shoot and inflorescence; b) floral leave; c) calyx; d) floral leave; e) leave. 2.-Teucrium x navarroi: a) habit; b) floral leave; c) calyx; d) floral leave; e) leave. 3.-Teucrium rivas-martinezii: a) shoot and inflorescence; b) floral leave; c) calyx; d) floral leave; e) leave. 

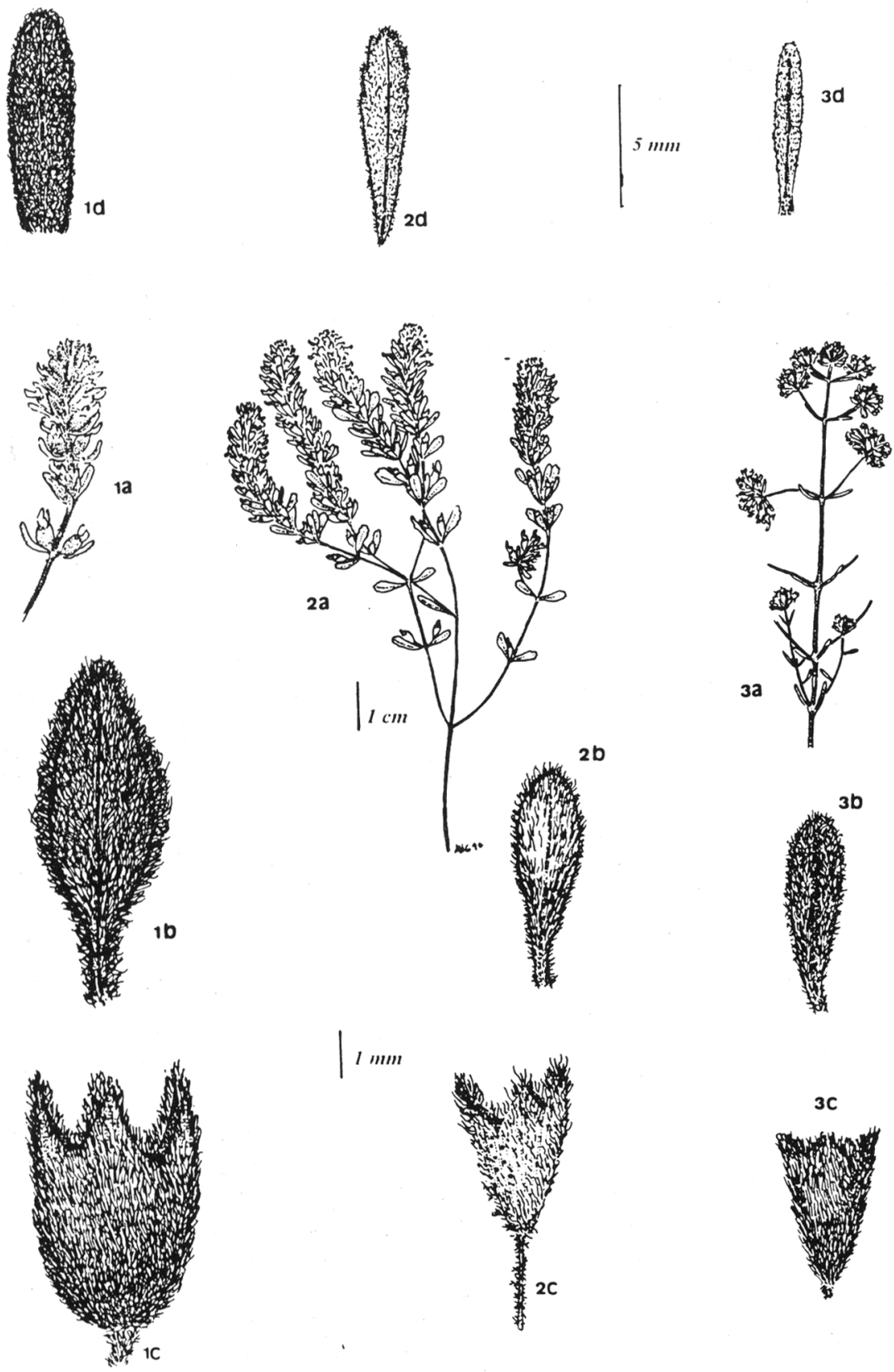

Figura 3. 1.-Teucrium gnaphalodes: a) inflorescencia; b) bracteola; c) cáliz; d) hoja. 2.-Teucrium $x$ conquense notosubsp. siyasense: a) inflorescencia; b) bracteola; c) cáliz; d) hoja. 3.-Teucrium capitatum subsp. gracillimun: a) inflorescencia; b) bracteola; c) cáliz; d) hoja. 1.-Teucrium gnaphalodes: a) inflorescence; b) floral leave; c) calyx; d) leave. 2.-Teucrium x conquense notosubsp. siyasense: a) inflorescence; b) floral leave; c) calyx; d) leave. 3.-Teucrium capitatum subsp. gracillimun: a) inflorescence; b) floral leave; c) calyx; d) leave. 
a quo tamen differt inflorescentia spiciforme, laxa. A T. gnaphalodi differt planta numquam tomentosa, folia linearia-oblonga, caulibus erecto-ascendentibus, indumento numquam cum pilis lanuginosis.

Habitat in collibus dumosis ad solum argilloso-calcareum in Regno Murcicu prope a Medina-Siyasa (Cieza).

Holotypus: MUB 47536. Murcia; Cieza, Charco Lentisco. 300 m 30SXH2938. 12-7-95. Cultivos abandonados. A. F. Carrillo \& Sánchez-Gómez. Isotypus: MGC 43000; VAL 35855 .

Planta cespitosa de hasta $30 \mathrm{~cm}$, leñosa en la base, ramas erecto-ascendentes con indumento grisáceo de pelos ramificados cortos. Hojas 15 × $3 \mathrm{~mm}$, oblongo-lineares, planas o ligeramente revolutas, crenadas en el tercio superior. Brácteas oblongo-lanceoladas, semejantes a las hojas. Inflorescencia en cabezuela terminal pauciflora, a veces espiciforme. Cáliz 3,5-4,5 mm, tubularovoideo, pedicelado con pedicelos de hasta 5 $\mathrm{mm}$, dientes triangular-obtusos. Corola 4,5-5 mm, blanca incluida en el tubo del cáliz.

T. $x$ conquense fue descrito a partir de material procedente de la Serranía de Cuenca, donde conviven $T$. capitatum subsp. capitatum y $T$. gnaphalodes. El aspecto externo es totalmente distinto al de la notosubespecie aquí descrita, ya que presenta porte de $T$. gnaphalodes e inflorescencia de $T$. capitatum (Crespo y Mateo, 1991). Sin embargo, el material murciano presenta un porte erectoascendente parecido a $T$. capitatum, e inflorescencias más desarrolladas, muy laxas, espiciformes. En la forma de las hojas, forma de las brácteas florales e indumento, que no llega a ser lanuginoso como T. gnaphalodes, el híbrido presenta caracteres intermedios entre los parentales; sin embargo, los cálices no son globosos como T. gnaphalodes y se aproximan más a T. capitatum. Es destacable en el híbrido la presencia de flores basales de la inflorescencia con largos pedicelos de hasta 5 $\mathrm{mm}$.

$\mathrm{El}$ areal en el que coinciden los parentales corresponde al sudeste Ibérico, no obstante, $T$. gnaphalodes suele estar en cotas más altas que T. capitatum subsp. gracillimum, por lo que el híbrido entre ambos taxones debe ser infrecuente (fig. 3).

\section{BIBLIOGRAFÍA}

CRESPO, B. M., \& G. MATEO -1991- New Spanish nothotaxa in the genus Teucrium L. (Lamiaceae). Fl. Medit., 1:195-203.

NAVARRO, T. -1988- Estudios biosistemáticos en el género Teucrium (Sección Polium (Mill.) Schreb. Subsect. Polium) en la Península Ibérica (Lamiaceae). Tesis doctoral Univ. Granada, Fac. de Ciencias, 2: 173+171 p.

NAVARRO, T. -1995-Revisión del género Teucrium L. sección Polium (Mill.) Schreb., (Lamiaceae) en la Península Ibérica y Baleares. Acta Bot. Malacitana, 20:173-265.

NAVARRO T. \& J.L. ROSÚA - 1990- Nomenclatural and taxonomic notes on the Teucrium Section Iberian Península. Candollea, 45 (2):581:589.

PUECH, S. -1984- Les Teucrium (Labiées) de la Sect. Polium du bassin Méditerranen occidental (France et Péninsule Ibérique). Naturalia Monsp., A5, $71 \mathrm{p}$.

SÁNCHEZ GÓMEZ, P. y F. ALCARAZ -1993Flora, Vegetación y Paisaje Vegetal de las Sierras de Segura Orientales. IEA. Albacete. $460 \mathrm{p}$.

Aceptado para su publicación en Julio de 1996

Dirección de los autores. P. Sánchez Gómez, A.F. Carrillo, E. Coy y A. Hernández: Dpto. de Biología Vegetal (Botánica), Facultad de Biología. Universidad de Murcia.30100 Campus de Espirnado. J. Güemes: Jardín Botánico de Valencia, Beato Gaspar Bono s/n. 46008 Valencia. 